\title{
A continuum approach to electrorheology
}

\author{
Yuri M. Shkel and Daniel J. Klingenberg ${ }^{\text {a) }}$ \\ Department of Chemical Engineering and Rheology Research Center, University \\ of Wisconsin, 1415 Engineering Drive, Madison, Wisconsin 53706
}

(Received 4 March 1999; final revision received 21 June 1999)

\begin{abstract}
Synopsis
An equilibrium thermodynamic approach is employed to derive a continuum-level expression for the electric field-induced stress in uniaxial anisotropic materials. Although this model is developed specifically to describe electrorheological and electrostrictive behavior of suspensions, it also applies to other uniaxial materials such as nonpolar nematic liquid crystals, biaxially oriented polymer films, and paper. This model introduces new electrostriction coefficients, which are material parameters that describe the strain dependence of the dielectric tensor as well as the field-induced stresses. An experimental technique for measuring the electrostriction parameters is outlined. An idealized microscopic model is presented to illustrate the relationships between microscopic parameters and the macroscopic electrostriction coefficients. The model is used to determine the stresses in common applications; predictions from the continuum approach agree with direct calculations from a microscopic approach of the normal stress and static shear modulus of electrorheological suspensions. (C) 1999 The Society of Rheology. [S0148-6055(99)01205-5]
\end{abstract}

\section{INTRODUCTION}

Electrostriction, defined as the field-induced deformation of a material, occurs in all materials [Stratton (1941)]. Electrorheology (ER), defined as the field-induced change in rheological properties, is typically only observed in anisotropic materials, such as suspensions of elongated particulate clusters dispersed in liquids or elastic media. Both phenomena are manifestations of field-induced electrostatic stresses. Electrostriction of isotropic materials is often described from a continuum perspective [Stratton (1941); Landau and Lifshitz (1984); Shkel and Klingenberg (1996)], while ER materials are commonly modeled from a microscopic perspective [see for example Brooks et al. (1986); Adriani and Gast (1988); Heyes and Melrose (1990); Whittle (1990); Klingenberg et al. (1991); McLeish et al. (1991); Bonnecaze and Brady (1992); Davis (1992); Halsey (1992); Kraynik et al. (1992); Lemaire et al. (1992); Tamura et al. (1993); Tao and Jiang (1994); Davis and Ginder (1995); Martin and Anderson (1996); Parthasarathy and Klingenberg (1996) and references therein], where the dynamics of the individual particles are related to macroscopic behavior. The goal of this paper is to develop a continuum, phenomenological model of ER suspensions that relates field-induced stresses to other measurable macroscopic properties. The resulting continuum model also extends the common description of electrostriction to anisotropic media.

We seek an equilibrium thermodynamic continuum description of electrorheological suspensions that employs the fewest assumptions about the material microstructure and

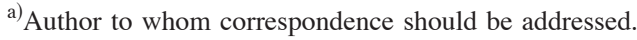


polarization mechanisms. ER composites are treated as continua with linear, homogeneous, and anisotropic dielectric properties without remnant polarization. The continuum description employs only macroscopic variables: the electric field $\mathbf{E}$, the dielectric displacement $\mathbf{D}$, the total stress $\boldsymbol{\sigma}$, and the strain $\mathbf{u}$. The only material properties that appear are the dielectric tensor $\boldsymbol{\epsilon}$ and the unit vector $\mathbf{d}$ that describes the material's anisotropy, which is the only structural information required. This definition also describes a variety of materials such as nonpolar nematic liquid crystals, oriented polymer films, and even ordinary paper. The total stress tensor, which contains mechanical and electrostatic contributions, is obtained directly from the variation of the free energy with deformation. The electrostatic portion of the stress tensor is thus expressed in terms of derivatives of the dielectric tensor components with respect to strain components. These derivatives are electrostriction parameters, which are material properties that can be measured experimentally.

There are several reasons for employing this approach. The material properties appearing in the continuum model are independent of the measuring geometry and technique, permitting direct comparison of results obtained on different systems and apparatuses. These material properties simultaneously describe dielectric and rheological behavior; thus rheological responses can be predicted from dielectric measurements, and vice versa. Furthermore, the rigorous thermodynamic continuum approach clearly reveals the number of independent measurements required to completely characterize a material. Once the material properties are known, the rheological and dielectric behavior for any deformation or geometry can be predicted. Finally, these predictions and relationships are obtained without detailed information about the microstructure and polarization phenomena.

Such a continuum model can be contrasted with microscopic models, commonly employed to study ER suspensions [reviewed in Parthasarathy and Klingenberg (1996)], where one must specify considerable microscopic detail. Particles are typically assumed to be spherical, interacting via electrically induced dipolar or higher order interactions, as well as some type of short-range repulsive forces. If one assumes a microstructure (e.g., the relative positions of all of the particles) and how it deforms locally under a macroscopic deformation, rheological properties can be calculated. Another approach is to assume an initial configuration and then determine the structure by numerically integrating the particles' equations of motion, which requires specifying the nature of the dissipative (hydrodynamic) forces acting on particles. Thus in these microscopic models, apparent rheological properties for specific deformations can be determined, subject to the numerous underlying assumptions. To quantitatively compare predictions with experimental results, properties introduced by the assumptions (such as particle size and shape, the particle polarizability, and the short-range forces) must be measured, which can be challenging. Furthermore, the apparent rheological properties predicted are not necessarily material properties, but rather functions of the system geometry and boundary conditions. In contrast, the continuum approach suggested here requires no such microscopic detail and focuses on strictly macroscopic material properties. The material properties depend, of course, on the microscopic details, but these parameters can be measured in independent experiments.

As an example, consider the thermodynamic continuum model for electrostatic stresses in isotropic materials [Stratton (1941); Landau and Lifshitz (1984)]. For small deformations, the dielectric tensor of an isotropic continuum is related to the deformation by

$$
\epsilon_{i k}=\epsilon^{0} \delta_{i k}+\alpha_{1} u_{i k}+\alpha_{2} u_{l l} \delta_{i k},
$$


where $\epsilon^{0}$ is the relative dielectric constant of the undeformed material, and $u_{i k}$ is the infinitesimal strain tensor. This phenomenological expression introduces the electrostriction parameters $\alpha_{1}$ and $\alpha_{2}$, which are thermodynamic material properties that can be measured experimentally [Shkel and Klingenberg (1996)]. The electrostatic stress resulting from an applied electric field, obtained from the variation of the free energy, is

$$
\sigma_{i k}^{\text {elect. }}=\frac{1}{2} \epsilon_{0}\left[\left(2 \epsilon^{0}-\alpha_{1}\right) E_{i} E_{k}-\left(\epsilon^{0}+\alpha_{2}\right) E^{2} \delta_{i k}\right]
$$

where $\epsilon_{0}$ is the permittivity of free space. To calculate the electrostatic force on a body within such a dielectric continuum, one integrates $\boldsymbol{\sigma}^{\text {elect. }} \cdot \mathbf{n}$ over the surface of the body. For liquid dielectrics, $\alpha_{1}=0$, and the procedure simplifies because the hydrostatic pressure increase caused by the electric field precisely cancels the contribution of $\alpha_{2}$ from Eq. (2) [Stratton (1941); Sanfeld (1968); Russel et al. (1989)]. The net electrostatic force exerted on a body in an isotropic liquid is thus described by the familiar Maxwell's stress tensor

$$
\sigma_{i k}^{\text {Maxwell }}=\epsilon_{0} \epsilon^{0}\left(E_{i} E_{k}-\frac{1}{2} E^{2} \delta_{i k}\right)
$$

For solid dielectrics, however, the electrostatic stress within the medium must be evaluated with Eq. (2). In fact, $\left|\alpha_{1}\right|$ and $\left|\alpha_{2}\right|$ are typically larger than $\epsilon^{0}$, and produce the dominant contribution to the electrostatic stress [Shkel and Klingenberg (1996), (1998)].

This example of the phenomenological description of electrostriction suggests a relation between electrostriction and electrorheology. Both are intimately related to, and arise from, electrostatic stresses. In the study of electrostriction, one is typically interested in a material's deformation caused by field-induced stresses, whereas in ER, one is typically interested in the change in the rheology - and thus the stress-caused by an electric field. The purpose of this article is to build on this relationship to develop a continuum level thermodynamic phenomenology for the stress and rheological properties of ER materials. By employing a formalism described by Landau and Lifshitz (1984), we obtain an expression for the field-induced stress in transverse isotropic materials. This essentially extends the continuum description of electrorheology published by Rosensweig (1995) by explicitly including the effect of electrostriction.

In Sec. II, we derive an equation for the electrostatic stress in an anisotropic, electrorheological continuum. We treat ER suspensions as transverse isotropic materials with a unique direction $\mathbf{d}$, but which are isotropic in the plane perpendicular to $\mathbf{d}$. This direction corresponds to the orientation of the columnar structures formed by the applied field. The strain-dependent dielectric tensor introduces seven electrostriction parameters, which are the anisotropic analogs of $\alpha_{1}$ and $\alpha_{2}$. The electrostatic stress is then determined from the free energy, and is written as a function of these dielectric parameters, as well as the dielectric tensor of the undeformed material $\epsilon_{i k}^{0}$, the orientation $\mathbf{d}$, and the electric field E. The general expression for the electrostatic stress simplifies for specific geometries, and examples for common cases are presented. We close Sec. II by describing an experimental procedure for measuring the electrostriction parameters.

In Sec. III, we illustrate the relationship between the continuum level description and microscopic features by examining an idealized microscopic model of electrorheological materials. An ER material is modeled as isolated chains of spherical particles. We first derive an expression for the strain-dependent dielectric tensor to extract the electrostriction parameters. Using these parameters, and the result for the electrostatic stress tensor from the continuum analysis, rheological properties of the idealized material are obtained. We then determine the same rheological properties by a more traditional route that 
does not employ the continuum representation, to show that the assumptions employed in the development in Sec. II are correct. This alternative route involves the direct summation of interparticle forces, and produces rheological properties identical to those obtained from the thermodynamic continuum model. The main points of this article are summarized in Sec. IV.

\section{FIELD INDUCED STRESSES IN ANISOTROPIC MEDIA}

\section{A. Phenomenological model}

Following Landau and Lifshitz (1984), we write the total stress in anisotropic media in terms of the free energy

$$
\sigma_{i k}=\tilde{F} \delta_{i k}+\left.\frac{\partial \tilde{F}}{\partial u_{i k}}\right|_{T, \mathbf{E}}+\frac{1}{2}\left(E_{i} D_{k}+E_{k} D_{i}\right),
$$

where $\sigma_{i k}=\sigma_{i k}^{\text {mech. }}+\sigma_{i k}^{\text {elect. }}$ is the mechanical (e.g., elastic) plus electrostatic stress, $\tilde{F}$ is the free energy per unit volume, and $u_{i j}$ is the strain tensor [for infinitesimal deformations, $u_{i j}=\left(\partial U_{i} / \partial x_{j}+\partial U_{j} / \partial x_{i}\right) / 2$, where $\mathbf{U}(\mathbf{x})$ is the displacement field]. The first term in Eq. (4) arises from volume variations, the second term arises from the change in free energy with deformation, and the third term arises from the effect of deformation and rotation of the structure on the electric field. The free energy per unit of volume of a polarizable material, $\tilde{F}\left(u_{i k}, T, \mathbf{E}\right)$, can be written as a contribution in the absence of a field, $F_{0} \equiv \tilde{F}\left(u_{i k}, T, \mathbf{E}=0\right)$, plus a contribution arising from the polarization

$$
\tilde{F}=F_{0}-\int_{0}^{E} \mathbf{D} \cdot \mathbf{d E}
$$

where

$$
\mathbf{D}=\epsilon_{0} \boldsymbol{\epsilon} \cdot \mathbf{E}
$$

and $\epsilon_{0}=8.8542 \times 10^{-12} \mathrm{~F} / \mathrm{m}$ is the permittivity of free space. The second order dielectric tensor $\boldsymbol{\epsilon}$ relates the dielectric displacement $\mathbf{D}$ to the electric field $\mathbf{E}$ in the material and is a function of the strain $u_{i k}$, temperature $T$, and electric field $\mathbf{E}$. Equations (5) and (6) are valid for essentially any material. In this article, we restrict attention to linearly polarizable media, where $\boldsymbol{\epsilon}$ is independent of field strength. In Eq. (6), $\boldsymbol{\epsilon}$ represents the measured dielectric tensor of the composite medium, which may be comprised from a variety of polarization mechanisms (e.g., from conductivity or permittivity differences between the disperse and continuous phases). One may also interpret $\boldsymbol{\epsilon}$ as that calculated exactly, including all multipole and multibody electric contributions.

The primary goal of this work is to obtain the electrostatic contribution to the total stress, $\boldsymbol{\sigma}^{\text {elect. }}=\boldsymbol{\sigma}-\boldsymbol{\sigma}^{\text {mech. }}$ for a certain class of anisotropic materials. The contribution $\boldsymbol{\sigma}^{\text {mech. }}$ is the stress in the absence of the electric field, which has been discussed extensively in the literature and will not be addressed here. According to Eqs. (4) and (5), calculating the electrostatic stress requires specifying the strain dependence of the polarization. The polarization of deformed isotropic materials has been described in terms of a strain-dependent dielectric tensor [Eq. (1)]. The strain dependence of the dielectric tensor for anisotropic materials can be written in a similar form [Landau and Lifshitz (1984)],

$$
\epsilon_{i k}=\epsilon_{i k}^{0}+\alpha_{i k l m} u_{l m}+\gamma_{i k l m} \omega_{l m}
$$




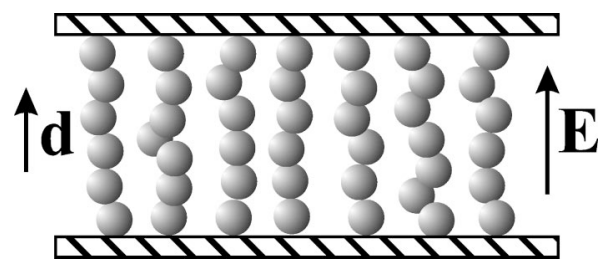

FIG. 1. Electrorheological liquid and solid heterogeneous systems are examples of transverse isotropic materials. Such systems have a distinct direction d, but are isotropic in the plane perpendicular to d. Rotation of transverse isotropic materials by any angle about $\mathbf{d}$ transposes the structure into itself.

where $\omega_{l m}=\left(\partial U_{l} / \partial x_{m}-\partial U_{m} / \partial x_{l}\right) / 2$ is the rotation tensor, and the superscript ${ }^{0}$ refers to the undeformed material. The fourth order electrostriction tensors $\alpha_{i k l m}$ and $\gamma_{i k l m}$ depend on the undeformed material's structure and the microscopic polarization phenomena.

Equation (7) represents the leading terms in a Taylor series expansion of $\epsilon_{i k}[\mathbf{U}(\mathbf{x})]$ for small deformations. Because the stress is related to $\partial \tilde{F} / \partial u_{i k}$, the linear terms describe the stress in a given state; higher-order terms are required to describe the general deformation-dependent stress. However, as shown below, one can still describe the deformation dependence of the stress for small shear deformations of ER composites using only the linear terms.

Because rigid rotations are treated explicitly in the last term in Eq. (4), the term $\gamma_{i k l m} \omega_{l m}$ need not appear in Eq. (7) [Landau and Lihfshitz (1984)]. Indeed, $\gamma_{i k l m}$ will not appear in the final expression for the stress. We retain this term, however, so that we may consider the effect of arbitrary deformations on the measured dielectric tensor.

Each of the tensors $\alpha_{i k l m}$ and $\gamma_{i k l m}$ contains 81 coefficients, but the number of independent coefficients depends on the symmetries possessed by the structure. The reduction for a particular type of anisotropy is presented below.

In this article, we consider the practically important type of anisotropy possessed by microstructures with a single distinct direction described by the unit vector d. Such uniaxial materials are labeled transverse isotropic. This type of anisotropy is considered here because it describes typical electro- and magnetorheological suspensions and elastomers, where particle columns or chains are oriented in a common direction, but where translational order is absent in the plane perpendicular to the columns (Fig. 1). Other examples of uniaxial materials with transverse isotropy include nematic liquid crystals, biaxially stretched films, and even ordinary paper (if one neglects the small differences between the machine and cross directions).

The dielectric properties of transverse isotropic materials differ in the $\mathbf{d}$ direction and in the plane perpendicular to $\mathbf{d}$. These materials have a second order dielectric tensor of the form

$$
\epsilon_{i k}=\epsilon^{\perp} \delta_{i k}+\left(\epsilon^{\|}-\epsilon^{\perp}\right) d_{i} d_{k},
$$

where $\epsilon^{\|}$and $\epsilon^{\perp}$ are the relative dielectric constants for probing fields applied parallel and perpendicular to $\mathbf{d}$, respectively.

The number of independent components in the tensors $\alpha_{i k l m}$ and $\gamma_{i k l m}$ are determined by the material symmetries. As shown in Appendix A, there are seven independent components for transverse isotropic materials. In this the case, the strain-dependent dielectric tensor can be written 


$$
\begin{aligned}
\epsilon_{i k}= & \epsilon_{i k}^{0}+\alpha_{1} u_{i k}+\alpha_{2} u_{l l} \delta_{i k}+\alpha_{3} u_{l m} d_{l}^{0} d_{m}^{0} \delta_{i k}+\alpha_{4}\left(u_{i l} d_{l}^{0} d_{k}^{0}+u_{k l} d_{l}^{0} d_{i}^{0}\right)+\alpha_{5} u_{l l} d_{i}^{0} d_{k}^{0} \\
& +\alpha_{6} u_{l m} d_{l}^{0} d_{m}^{0} d_{i}^{0} d_{k}^{0}+\gamma_{1}\left(\omega_{i l} d_{l}^{0} d_{k}^{0}+\omega_{k l} d_{l}^{0} d_{i}^{0}\right) .
\end{aligned}
$$

Equation (9) introduces seven phenomenological electrostriction coefficients $\alpha_{1}-\alpha_{6}$, and $\gamma_{1}$. These material properties are scalar functions of thermodynamic variables, such as the temperature, the electric field magnitude, and the density. The dielectric constants $\epsilon^{\|}$ and $\epsilon^{\perp}$ of the undeformed material are well-known material parameters, which can be measured by a variety of experimental methods and related to the material microstructure through a number of theoretical models. The parameters $\alpha_{1}$ and $\alpha_{2}$ were first introduced by Stratton (1941) and Landau and Lifshitz (1984) for isotropic materials. Experimental methods for measuring these parameters and microscopic models for predicting these parameters have been discussed by Shkel and Klingenberg $(1996,1998)$. The parameters $\alpha_{3}-\alpha_{6}$, and $\gamma_{1}$ are new and have not been studied before.

Consider the stress in a transverse isotropic material with the vector $\mathbf{d}$ oriented arbitrarily with respect to the applied electric field $\mathbf{E}=E \mathbf{e}$, where $\mathbf{e}$ is a unit vector in the direction of the electric field. The stress tensor is obtained by first substituting Eq. (9) for the strain-dependent dielectric tensor along with Eq. (6) into Eq. (5) for the free energy, and then determining the stress via Eq. (4). The result for the electrostatic contribution to the stress tensor for a material with orientation $\mathbf{d}$ is

$$
\begin{aligned}
\sigma_{i k}^{\text {elect. }}= & \frac{1}{2} \epsilon_{0} E^{2}\left[\epsilon^{\perp}\left(2 e_{i} e_{k}-\delta_{i k}\right)+\left(\epsilon^{\|}-\epsilon^{\perp}\right)\left(e_{i} d_{k}+e_{k} d_{i}-e_{m} d_{m} \delta_{i k}\right) d_{l} e_{l}-\alpha_{1} e_{i} e_{k}\right. \\
& \left.-\alpha_{2} \delta_{i k}-\alpha_{3} d_{i} d_{k}-\alpha_{4}\left(e_{i} d_{k}+e_{k} d_{i}\right) e_{l} d_{l}-\alpha_{5}\left(e_{l} d_{l}\right)^{2} \delta_{i k}-\alpha_{6}\left(e_{l} d_{l}\right)^{2} d_{i} d_{k}\right] .
\end{aligned}
$$

The stress tensor depends on eight phenomenological material parameters: the dielectric constants $\epsilon^{\|}$and $\epsilon^{\perp}$, and the electrostriction parameters $\alpha_{1}-\alpha_{6}$. These parameters are properties of the structure in its current state (d), and must be measured experimentally (Sec. II D), or predicted from a microscopic model of the material structure (Sec. III). As expected, Eq. (10) reduces to Eq. (2) for the electrostatic stress tensor in isotropic media, where $\epsilon^{\|}=\epsilon^{\perp}$ and $\alpha_{3}-\alpha_{6}=0$.

Equation (4) for the total stress is symmetric. This is expected because the model does not include a vector density of body couples exerted by external sources, and thus according to angular momentum conservation the stress must be symmetric [Sedov (1971); Landau and Lifshitz (1984); Rosensweig (1995)]. The total stress is divided into a mechanical stress $\sigma_{i k}^{\text {mech. }}=\sigma_{i k}(E=0)$ plus the electrostatic contribution. Since the mechanical stress is symmetric, the electrostatic contribution to the stress [Eq. (10)] is also symmetric. The division of the total stress is arbitrary, as long as the sum of the parts results in a symmetric stress tensor.

The division of the total stress into electrostatic and mechanical contributions proposed in this article is different than that employed by Rosensweig (1995) in an analysis of linearly magnetizable media. In terms of the analogous electrostatic quantities, Rosensweig defined the field-dependent contribution as

$$
\sigma_{i j}^{\text {Rosen. }}=D_{i} E_{j}-\frac{1}{2} \epsilon_{0} E^{2} \delta_{i j},
$$

neglecting electrostriction. The remaining contribution to the stress was defined as the elastic contribution. Both contributions are asymmetric, while their sum is symmetric. However, since the total stress must be symmetric in the absence of an applied field, the elastic contribution defined by Rosensweig must contain field-dependent terms, which 

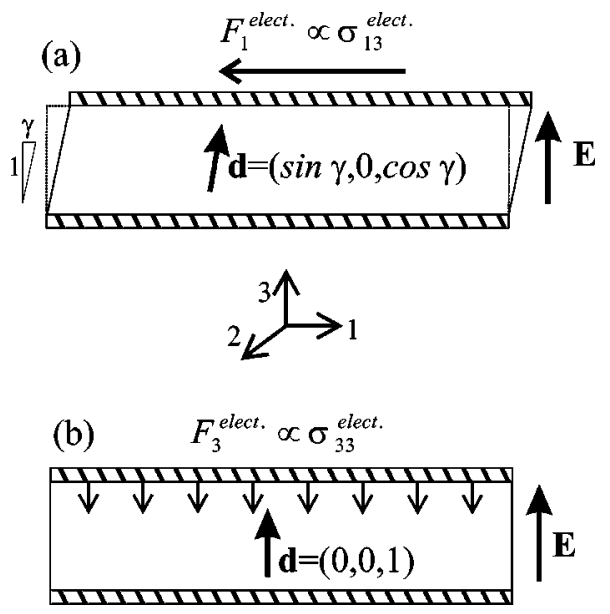

FIG. 2. Schematic diagrams of two common experimental configurations for ER suspensions: (a) small amplitude shear deformation, and (b) the normal stress caused by an electric field.

were ignored in the subsequent analysis. The thermodynamic derivation in the present article reveals the missing contributions to the electrostatic stress, which are contained in Eq. (10).

\section{B. Example solutions for field-induced stresses}

Equation (10) describes the stress of arbitrary transverse isotropic structures in arbitrary states of (infinitesimal) deformation and for arbitrary orientations of the structure relative to the electric field. Here we illustrate simplifications of stress components for a few common situations, illustrated schematically in Fig. 2.

\section{Simple shear}

Consider a material with the anisotropy vector initially aligned with the electric field $\mathbf{E}=(0,0, E)$, and subjected to a small simple shear deformation where the nonzero components of the strain tensor are $u_{13}=u_{31}=\gamma / 2$. After deformation, the anisotropy vector is $\mathbf{d}=(\sin \gamma, 0, \cos \gamma)$. To leading order in $\gamma$, Eq. (10) for the shear stress simplifies to

$$
\sigma_{13}^{\text {elect. }}=\epsilon_{0} \frac{E^{2}}{2} \gamma\left\{\left(\epsilon^{\|}-\epsilon^{\perp}\right)-\tilde{\alpha}_{3}\right\}
$$

where $\tilde{\alpha}_{3} \equiv \alpha_{3}+\alpha_{4}+\alpha_{6}$. The phenomenological parameters are functions of the structure in the deformed state. In Sec. III, a microscopic model is employed to calculate the phenomenological parameters. For simple shear, the leading order variation of the parameters with strain is $O\left(\gamma^{2}\right)$, and thus the parameters in Eq. (11) can be evaluated for the undeformed structure.

\section{Normal stress}

Consider a material with $\mathbf{d}=(0,0,1)$ directed along the field. The normal stress component $\sigma_{33}^{\text {elect. }}$ from Eq. (10) simplifies to

$$
\sigma_{33}^{\text {elect. }}=\epsilon_{0} \frac{E^{2}}{2}\left\{\epsilon^{\|}-\left(\tilde{\alpha}_{1}+\tilde{\alpha}_{2}+\tilde{\alpha}_{3}\right)\right\},
$$


ëúwhere $\tilde{\alpha}_{1} \equiv \alpha_{1}+\alpha_{4}$ and $\tilde{\alpha}_{2} \equiv \alpha_{2}+\alpha_{5}$. Again, the parameters are evaluated for the current state of the structure.

Both Eqs. (11) and (12) express the relevant stress components in the simple, intuitive form $\sigma_{i k}^{\text {elect. }}=K_{i k} E^{2}$. The thermodynamics formalism relates the proportionality constants $K_{i k}$ to measurable dielectric properties.

\section{Relations between dielectric parameters}

The number of dielectric parameters that must be measured experimentally or predicted from microscopic models can be reduced by interrelating some of them. Consider the rigid rotation of a structure from an initial orientation $\mathbf{d}^{0}=(0,0,1)$ to a new orientation $\mathbf{d}$, about an axis perpendicular to $\mathbf{d}^{0}$. The dielectric tensor in the rotated state is related to the initial dielectric tensor by Eq. (9), which reduces to

$$
\epsilon_{i k}=\epsilon^{\perp} \delta_{i k}+\left(\epsilon^{\|}-\epsilon^{\perp}\right) d_{i}^{0} d_{k}^{0}+\gamma_{1}\left(\omega_{i l} d_{l}^{0} d_{k}^{0}+\omega_{k l} d_{l}^{0} d_{i}^{0}\right) .
$$

The eigenvalues $\epsilon^{\|}$and $\epsilon^{\perp}$ are not affected by the rotation, and thus the dielectric tensor may also be expressed in terms of the rotated state $\mathbf{d}$ by Eq. (8). Substituting $d_{i}=d_{i}^{0}$ $+\omega_{i j} d_{j}^{0}$ into Eq. (8) and equating with Eq. (13) above gives

$$
\gamma_{1}=\epsilon^{\|}-\epsilon^{\perp} \text {. }
$$

This result is valid for any transverse isotropic structure.

Next consider a structure initially oriented with $\mathbf{d}^{0}=(0,0,1)$ subjected to simple shear: $\mathbf{U}(\mathbf{x})=(\gamma z, 0,0)$. The dielectric tensor component $\epsilon_{13}$ can be obtained from Eq. (9) as

$$
\epsilon_{13}^{\text {shear }}=\frac{1}{2} \gamma\left(\alpha_{1}+\alpha_{4}+\gamma_{1}\right) .
$$

For the same initial structure exposed to rigid rotation about the $y$ axis by an angle $\gamma, \epsilon_{13}$ becomes

$$
\epsilon_{13}^{\text {rot. }}=\gamma \gamma_{1}
$$

Assuming affine deformation, particle displacements for these two deformations differ by $O\left(\gamma^{2}\right)$, and thus $\epsilon_{13}^{\text {shear }}=\epsilon_{13}^{\text {rot. }}$ to $O(\gamma)$. Equating Eqs. (15) and (16), we obtain

$$
\alpha_{1}+\alpha_{4}=\epsilon^{\natural}-\epsilon^{\perp} .
$$

\section{Obtaining the dielectric parameters from experimental measurements}

The macroscopic model developed in Sec. II contains nine phenomenological parameters: $\epsilon^{\|}, \epsilon^{\perp}, \alpha_{(i)}(i=1 \ldots 6)$, and $\gamma_{1}$. The dielectric coefficients of the undeformed material, $\epsilon^{\|}$and $\epsilon^{\perp}$, can be obtained from standard dielectric measurements with the probing electric field oriented parallel and perpendicular to d, respectively. As discussed in Sec. II C, $\gamma_{1}$ and $\alpha_{1}+\alpha_{4}$ are both equal to $\epsilon^{\|}-\epsilon^{\perp}$. This leaves five dielectric parameters (or combinations thereof) that must be determined independently to utilize the macroscopic continuum model. The remaining parameters can be determined experimentally by measuring the deformation dependence of the dielectric tensor under different strain fields $u_{i k}$. Such an experimental technique has been described by Shkel and Klingenberg (1996).

Consider deformations with diagonal strain tensors of a material initially oriented with $\mathbf{d}^{0}=(0,0,1)$. For such deformations the structure is not rotated, and thus $\boldsymbol{\omega} \equiv \mathbf{0}$ and 
TABLE I. Summary of the measured component of the dielectric tensor and the relative values of the strain components required to extract the dielectric parameters $\alpha_{1}, \alpha_{2}, \alpha_{3}, \alpha_{5}$ and $\alpha_{6}$.

\begin{tabular}{clc}
\hline $\begin{array}{c}\text { Dielectric tensor } \\
\text { component measured }\end{array}$ & \multicolumn{1}{c}{$\begin{array}{c}\text { Strain tensor } \\
\text { components }\end{array}$} & $\begin{array}{c}\text { Parameter } \\
\text { obtained }\end{array}$ \\
\hline$\epsilon_{11}$ & $u_{11}=-u_{22} \neq 0, u_{33}=0$ & $\alpha_{1}$ \\
$\epsilon_{11}$ & $u_{11}=u_{33}=0, u_{22} \neq 0$ & $\alpha_{2}$ \\
$\epsilon_{11}$ & $u_{11}=0, u_{22}=-u_{33} \neq 0$ & $\alpha_{3}$ \\
$\epsilon_{33}$ & $u_{11}+u_{22} \neq 0, u_{33}=0$ & $\alpha_{5}$ \\
$\epsilon_{33}$ & $u_{11}+u_{22}=-u_{33} \neq 0$ & $\alpha_{6}$ \\
\hline \hline
\end{tabular}

$\mathbf{d}=\mathbf{d}^{0}$. From Eqs. (8) and (9), both $\epsilon_{i k}^{0}$ and $\epsilon_{i k}$ are diagonal as well. The dielectric tensor components $\epsilon_{11}$ and $\epsilon_{33}$ reduce to

$$
\begin{gathered}
\epsilon_{11}=\epsilon^{\perp}+\alpha_{1} u_{11}+\alpha_{2} u_{k k}+\alpha_{3} u_{33}, \\
\epsilon_{33}=\epsilon^{\|}+\left(\alpha_{1}+\alpha_{3}+2 \alpha_{4}+\alpha_{6}\right) u_{33}+\left(\alpha_{2}+\alpha_{5}\right) u_{k k} .
\end{gathered}
$$

The relative magnitudes of the strain components $u_{11}, u_{22}$ and $u_{33}$ can be altered by varying the mechanical boundary conditions during deformation. Thus by measuring the variation in the dielectric tensor components for different boundary conditions, the remaining dielectric parameters can be extracted via Eqs. (18) and (19). The measured dielectric tensor component and the values of the strain tensor components required to extract the various dielectric parameters are summarized in Table I. This approach of examining the change in the dielectric tensor with deformation to extract the dielectric parameters may also be employed to predict the dielectric parameters from microscopic models, as described in Sec. III.

\section{AN ILLUSTRATIVE MICROSCOPIC MODEL}

Electrostriction parameters can either be measured, as described above, or calculated from a microscopic model. In this section we illustrate one such microscopic model, namely isolated particle chains, to demonstrate how the electrostriction coefficients can be obtained from microscopic considerations. With this model, we first determine the strain-dependent dielectric tensor, from which the dielectric parameters can be extracted as described in Sec. II, and the stress can be predicted via Eq. (10). Using the same microscopic model, we then determine the stress by directly calculating forces transmitted across boundaries, without any consideration of the phenomenological model, to verify the assumptions employed in the phenomenological derivation of the stress tensor [Eq. (10)].

We consider the material as a composite of identical polarizable spheres in a dielectric medium (relative dielectric constant $\boldsymbol{\epsilon}_{c}$ ). The sphere polarizability is $\alpha$ $=(\pi / 2) \epsilon_{0} \epsilon_{c} \sigma^{3} \beta$, where $\sigma$ is the sphere diameter, and $\beta$ is the relative dielectric constant. For nonconducting phases, $\beta=\left(\epsilon_{p}-\epsilon_{c}\right) /\left(\epsilon_{p}+2 \epsilon_{c}\right)$ where $\epsilon_{p}$ is the relative dielectric constant of the spheres; for conducting phases in dc electric fields, $\beta=\left(\Sigma_{p}\right.$ $\left.-\Sigma_{c}\right) /\left(\Sigma_{p}+2 \Sigma_{c}\right)$, where $\Sigma_{p}$ and $\Sigma_{c}$ are the conductivities of the particulate and continuous phases, respectively.

The particles are arranged in chains with equal pair separations (Fig. 3). The chains are sufficiently far apart such that the particles in one chain only affect the polarization of the spheres in the same chain. Furthermore, quadrupole and higher-order disturbance 


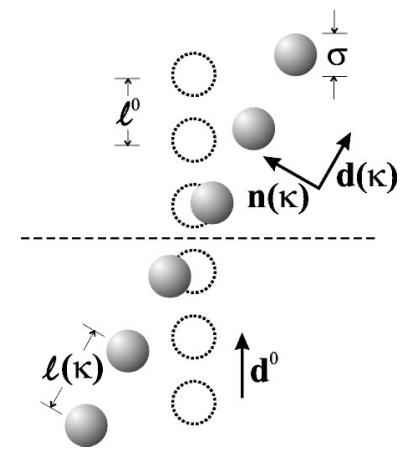

FIG. 3. A representative chain of spheres in a transverse isotropic structure deformed by a displacement gradient $\kappa_{i j}$.

fields are neglected. The dipole moment on each sphere, proportional to the local electric field, is determined self-consistently. This polarization model has been used numerous times [Jeffrey (1973); Adriani and Gast (1988); Tao and Sun (1991); Davis (1992); Xu and Hass (1993)]. However, treating only dipole interactions significantly underpredicts field-induced stresses [Bonnecaze and Brady (1992); Parthasarathy and Klingenberg (1996)]. We have chosen this polarization model because it has been extensively explored by others and is thus convenient for comparison. In addition, this model constitutes the simplest illustrative example in which the thermodynamic coefficients can be evaluated from microscopic considerations.

Consider the structure illustrated in Fig. 3. Before deformation, the chain is oriented in the $\mathbf{d}^{0}$ direction, and the spheres are separated by a distance $\ell^{0}$. The composite, and thus each chain, is deformed by a displacement gradient $\kappa_{i j}$. After deformation, the chains are oriented in the $\mathbf{d}(\boldsymbol{\kappa})$ direction, and the spheres are separated by the distance $\ell(\boldsymbol{\kappa})$.

\section{A. Strain-dependent dielectric tensor}

The electrostriction parameters are determined by calculating the dielectric tensor in the deformed state, and then relating this tensor to the undeformed structure. The dielectric tensor is related to the mean dipole moment $\mu$ via the polarization

$$
\epsilon_{0}\left(\epsilon_{i k}-\epsilon_{c} \delta_{i k}\right) E_{k}=n \mu_{i} .
$$

The mean dipole moment is equivalent to the moment of each sphere, because all spheres are equivalent.

The dipole moment of a reference sphere $k$ equals the sphere polarizability $\alpha$ multiplied by the local field, which is the applied field plus the disturbance fields generated by all the other dipoles

$$
\mu_{i}=\alpha\left(E_{i}-\sum_{l \neq k} T_{i j}\left(\mathbf{r}^{(k l)}\right) \mu_{j}\right),
$$

where the sum is over all spheres $l$ in a chain, excluding reference sphere $k, \mathbf{r}^{(k l)}$ $\equiv \mathbf{r}^{(l)}-\mathbf{r}^{(k)},\left(\mathbf{r}^{(l)}\right.$ is the position of sphere $\left.l\right)$ and

$$
T_{i j}(\mathbf{r})=\frac{1}{4 \pi \epsilon_{0} \epsilon_{c}}\left(\frac{\delta_{i j}}{r^{3}}-3 \frac{r_{i} r_{j}}{r^{5}}\right)
$$

is the dipole interaction tensor. 
The dipole moment can be separated into components parallel and perpendicular to the chain,

$$
\mu_{i}=\mu^{\|} d_{i}+\mu^{\perp} n_{i}
$$

Substituting this into Eq. (21), the components can be resolved as

$$
\begin{gathered}
\mu^{\|}=\alpha \lambda^{\|} E^{\|}, \\
\mu^{\perp}=\alpha \lambda^{\perp} E^{\perp},
\end{gathered}
$$

where $E^{\|}$and $E^{\perp}$ are the components of the applied field parallel and perpendicular to the chain, respectively. The factors $\lambda^{\|}$and $\lambda^{\perp}$ describe the enhancement of the local electric field strength relative to the applied field strength for probing fields applied parallel or perpendicular to the chain, respectively, and are expressed

$$
\begin{aligned}
\lambda^{\|} & =\frac{1}{1-\frac{1}{2} \beta \zeta(3)(\sigma / \ell)^{3}}, \\
\lambda^{\perp} & =\frac{1}{1+\frac{1}{4} \beta \zeta(3)(\sigma / \ell)^{3}},
\end{aligned}
$$

where $\zeta(3)=\sum_{n=1}^{\infty} n^{-3} \approx 1.20206$. Substituting these results into Eq. (20) yields the components of the dielectric tensor

$$
\begin{gathered}
\epsilon^{\|}=\epsilon_{c}+\frac{n \alpha}{\epsilon_{0}} \lambda^{\|}, \\
\epsilon^{\perp}=\epsilon_{c}+\frac{n \alpha}{\epsilon_{0}} \lambda^{\perp} .
\end{gathered}
$$

The electrostriction coefficients can be obtained by simply determining the deformation dependence of $\epsilon^{\|}$and $\epsilon^{\perp}$. The relevant deformation-dependent quantities are the sphere pair separation $\ell(\boldsymbol{\kappa})$, the orientation vector $\mathbf{d}(\boldsymbol{\kappa})$, and the sphere number density $n(\boldsymbol{\kappa})$ (see Fig. 3),

$$
\begin{gathered}
\ell(\boldsymbol{\kappa})=\ell^{0} \sqrt{1+2 \kappa_{i j} d_{i}^{0} d_{j}^{0}+\kappa_{i j} \kappa_{i k} d_{j}^{0} d_{k}^{0}}, \\
d_{i}(\boldsymbol{\kappa})=\frac{\ell^{0}}{\ell(\boldsymbol{\kappa})}\left(d_{i}^{0}+\kappa_{i j} d_{j}^{0}\right), \\
n(\boldsymbol{\kappa})=\frac{n^{0}}{1+\kappa_{i i}+\frac{1}{2}\left(\kappa_{i i}^{2}-\kappa_{i j} \kappa_{j i}\right)} .
\end{gathered}
$$

Substituting these expressions into Eqs. (28) and (29), substituting these results into Eq. (8), and expanding for small $\kappa_{i j}$ yields the following expression for the dielectric tensor in terms of the undeformed structure $\left(\mathbf{d}^{0}\right)$ : 


$$
\begin{aligned}
\epsilon_{i k}= & \epsilon_{i k}^{0}+\frac{\alpha n^{0}}{\epsilon_{0}}\left\{\left(\lambda^{\|, 0}-\lambda^{\perp, 0}\right)\left(d_{i}^{0} d_{l}^{0} \omega_{k l}+d_{k}^{0} d_{l}^{0} \omega_{i l}\right)+\left(\lambda^{\|, 0}-\lambda^{\perp, 0}\right)\left(d_{i}^{0} d_{l}^{0} u_{k l}+d_{k}^{0} d_{l}^{0} u_{i l}\right)\right. \\
& -\lambda^{\perp, 0} \delta_{i k} u_{l l}+\frac{3}{2} \xi\left(\lambda^{\perp, 0}\right)^{2} \delta_{i k} d_{l}^{0} d_{m}^{0} u_{l m}-\left(\lambda^{\|, 0}-\lambda^{\perp, 0}\right) d_{i}^{0} d_{k}^{0} u_{l l} \\
& \left.-\left[2\left(\lambda^{\|, 0}-\lambda^{\perp, 0}\right)+3 \xi\left(\left(\lambda^{\|, 0}\right)^{2}+\frac{1}{2}\left(\lambda^{\perp, 0}\right)^{2}\right)\right] d_{i}^{0} d_{k}^{0} d_{l}^{0} d_{m}^{0} u_{l m}\right\},
\end{aligned}
$$

where

$$
\xi=\frac{1}{2} \beta \zeta(3)\left(\frac{\sigma}{\ell^{0}}\right)^{3} .
$$

The electrostriction coefficients may be extracted by considering the effects of different deformations on $\epsilon_{i k}$ as described in Sec. II, or by direct comparison of Eq. (33) with Eq. (9). The coefficients are

$$
\begin{gathered}
\alpha_{1}=0, \\
\alpha_{2}=-\left(\epsilon^{\perp}-\epsilon_{c}\right), \\
\alpha_{3}=3\left(\epsilon^{\perp}-\epsilon_{c}\right) \frac{\frac{1}{2} \xi}{1+\frac{1}{2} \xi}, \\
\alpha_{4}=\epsilon^{\|}-\epsilon^{\perp}, \\
\alpha_{5}=-\left(\epsilon^{\|}-\epsilon^{\perp}\right), \\
\alpha_{6}=-2\left(\epsilon^{\|}-\epsilon^{\perp}\right)-3\left[\left(\epsilon^{\|}-\epsilon_{c}\right) \frac{\xi}{1-\xi}+\left(\epsilon^{\perp}-\epsilon_{c}\right) \frac{\frac{1}{2} \xi}{1+\frac{1}{2} \xi}\right], \\
\gamma_{1}=\epsilon^{\|}-\epsilon^{\perp} .
\end{gathered}
$$

For a structure initially aligned with the electric field, the shear stress can be obtained by substituting the appropriate electrostriction coefficients listed above into Eq. (11). The resulting shear modulus is

$$
\begin{aligned}
G^{\text {elect. }}=\sigma_{13}^{\text {elect. } / \gamma} & =3 \phi \epsilon_{0} \epsilon_{c} \beta E^{2}\left(\frac{\xi}{1-\xi}+\frac{\frac{1}{2} \xi}{1+\frac{1}{2} \xi}\right)+\frac{3}{2} \phi \epsilon_{0} \epsilon_{c} \beta E^{2} \frac{3 \xi}{(1-\xi)^{2}} \\
& =\epsilon_{0}\left(\epsilon^{\|}-\epsilon^{\perp}\right) E^{2}+\epsilon_{0} \frac{\left(\epsilon^{\|}-\epsilon_{c}\right)^{2}}{\epsilon_{c}} \frac{3 \zeta(3)}{2 \pi n\left(\ell^{0}\right)^{3}} E^{2} .
\end{aligned}
$$

Equation (41) is identical to that obtained by Davis (1992). Equation (42) shows that the modulus contains two contributions. The first term is equivalent to $-(\mathbf{P} \times \mathbf{E})_{2}$, where $\mathbf{P}$ is the macroscopic polarization. This contribution arises because the polarization is not aligned with the applied field when the structure is sheared. The second term in Eq. (42) arises from chain stretching (i.e., electrostriction), and is of equal or greater magnitude than the first term [Davis (1992)]. 
The normal stress for a structure aligned with the applied electric field may be evaluated by substituting the appropriate electrostriction coefficients listed above into Eq. (12). The result can be written in the form

$$
\sigma_{33}^{\text {elect. }}=\frac{1}{2} \epsilon_{0} \epsilon^{\|} E^{2}+\frac{1}{2} \epsilon_{0}\left(\epsilon^{\|}-\epsilon_{c}\right) E^{2}+\frac{3}{2} \phi \epsilon_{0} \epsilon_{c} \beta E^{2} \frac{3 \xi}{(1-\xi)^{2}} .
$$

In Sec. III B, $G^{\text {'elect. }}$ and $\sigma_{33}^{\text {elect. }}$ are obtained by a different approach that employs the direct calculation of interparticle forces.

\section{B. Direct calculation of stress tensor components}

Here we calculate the shear and normal stresses using a different approach that does not employ the continuum stress tensor expression. Instead, the stress components are obtained by the direct summation of the appropriate interparticle forces.

To evaluate the shear stress, consider again the same microscopic model employed in the previous expression, with the chain structure deformed from its original state (where it is aligned with the electric field). Specifically, we consider simple shear with the displacement field $\mathbf{U}(\mathbf{x})=\left(\gamma x_{3}, 0,0\right)$. The shear stress is

$$
\sigma_{13}^{\text {elect. }}=N F_{x}^{\text {chain }}
$$

where $N=6 \phi \ell^{0} /\left(\pi \sigma^{3}\right)$ is the number of chains per unit area in a plane normal to $\mathbf{d}^{0}$ and $F_{x}^{\text {chain }}$ is the $x$ component of the force exerted by the top half of a chain (spheres $k$ above the plane) on the bottom half of a chain (spheres $l$ below the plane). The quantity $F_{x}^{\text {chain }}$ is the pairwise summation of the interparticle forces

$$
F_{x}^{\text {chain }}=\sum_{k=1}^{\infty} \sum_{i=1}^{\infty} \mathbf{F}^{(k l)}\left(\mathbf{r}^{(k l)}\right) \cdot \mathbf{e}_{x}
$$

where $\mathbf{F}^{(k l)}\left(\mathbf{r}^{(k l)}\right)$ is the force exerted by sphere $k$ at $\mathbf{r}^{(k)}$ on sphere $l$ at $\mathbf{r}^{(l)}$.

Again employing the point-dipole approximation, the electrostatic interparticle force is

$$
\begin{aligned}
\mathbf{F}^{(k l)} & =\left.\boldsymbol{\mu} \cdot \boldsymbol{\nabla} \mathbf{E}^{(k)}\right|_{\mathbf{x}=\mathbf{r}^{(l)}} \\
& =\frac{1}{4 \pi \boldsymbol{\epsilon}_{0} \boldsymbol{\epsilon}_{c}}\left[-6 \frac{\left(\mathbf{r}^{(k l)} \cdot \boldsymbol{\mu}\right) \boldsymbol{\mu}}{r^{(k l)^{5}}}-3 \frac{|\boldsymbol{\mu}|^{2} \mathbf{r}^{(k l)}}{r^{(k l)^{5}}}+15 \frac{\left(\mathbf{r}^{(k l)} \cdot \boldsymbol{\mu}\right)^{2} \mathbf{r}^{(k l)}}{r^{(k l)^{7}}}\right],
\end{aligned}
$$

where $\boldsymbol{\nabla} \mathbf{E}^{(k)} \mid \mathbf{x}=\mathbf{r}^{(l)}$ is the gradient of the disturbance field caused by sphere $k$ evaluated at the center of sphere $l, r^{(k l)}=\left|\mathbf{r}^{(k l)}\right|$, and $\boldsymbol{\mu}$ is the dipole moment obtained selfconsistently in Sec. II A.

Substituting Eq. (46) into Eq. (45), and then the result into Eq. (44), and expanding for small $\gamma$ yields

$$
G^{\text {elect. }}=3 \phi \epsilon_{0} \epsilon_{c} \beta E^{2}\left(\frac{\xi}{1-\xi}+\frac{\frac{1}{2} \xi}{1+\frac{1}{2} \xi}\right)+\frac{3}{2} \phi \epsilon_{0} \epsilon_{c} \beta E^{2} \frac{3 \xi}{(1-\xi)^{2}},
$$

which is identical to the result obtained from the thermodynamic approach.

To calculate the normal stress, three contributions must be accounted for:

(i) The force exerted by the dipoles above the plane on the dipoles below the plane, calculated in a manner similar to that described above for the shear stress. This contribution to the normal stress is $(3 / 2) \phi \epsilon_{0} \epsilon_{c} \beta E^{2}\left[3 \xi /(1-\xi)^{2}\right]$. 
(ii) The force exerted by the dipoles above the plane on the dielectric matrix below the plane. This contribution to the normal stress is $(1 / 2) \epsilon_{0}\left(\epsilon^{\|}-\epsilon_{c}\right) E^{2}$.

(iii) The Coulombic force exerted by the free charge on the top electrode on the free charge on the bottom electrode. This contribution to the normal stress is $(1 / 2) \epsilon_{0} \epsilon^{\|} E^{2}$.

Adding these three contributions together gives the right side of Eq. (43). This direct calculation illuminates the origin of each term in Eq. (43). However, in the direct calculation, these terms must be identified explicitly, whereas they arise naturally when evaluating the stress via the electrostriction coefficients. We therefore feel that our evaluation of the electroactivity in terms of the dielectric properties is more straightforward, particularly when the systems become more complicated (e.g., when they include interacting chains, amorphous anisotropic structures, different polarization phenomena, etc.).

\section{CONCLUSION}

We have derived an expression for the electric field-induced stress in electrorheological suspensions employing only measurable macroscopic properties. This is achieved by defining a strain-dependent dielectric tensor that introduces electrostriction coefficients, and by employing an equilibrium thermodynamics formalism. The analysis suggests that electrorheology and electrostriction are essentially the same phenomenon, and that ER responses can be realized without dissipative mechanisms. The model provides a foundation for distinguishing equilibrium and nonequilibrium processes in ER suspensions.

The strain-dependent dielectric tensor introduces seven electrostriction parameters. A procedure is presented for measuring these parameters experimentally, and an idealized microscopic model illustrates how one can predict the parameters theoretically. Predictions from the microscopic model agree with direct calculations.

There are several benefits to employing a continuum approach. The electrostriction coefficients are independent of the measuring geometry and technique, permitting direct comparison of results obtained on different systems and apparatuses. These coefficients simultaneously describe dielectric and rheological behavior; thus rheological responses can be predicted from dielectric measurements, and vice versa. These predictions and relationships are obtained without detailed information about the microstructure and polarization phenomena. We believe that the overriding theme of interpreting rheological properties in terms of macroscopic dielectric properties may offer new approaches for engineering new materials. For example, one can consider how particle morphology or system anisotropy will affect the dielectric properties that govern the rheology (e.g., $\tilde{\alpha}_{1}, \tilde{\alpha}_{2}, \tilde{\alpha}_{3}$, etc.) as opposed to the apparent, apparatus-dependent rheological response itself.

This article represents a preliminary step toward developing a complete continuum representation of electroactivity; numerous aspects deserve further attention. Experimental data for the electrostriction parameters for different systems should be obtained and the subsequently predicted stresses compared with rheological measurements. The straindependent dielectric tensor should be extended to higher order in strain to obtain the strain dependence of the electrostatic stress, to evaluate electrorheological properties more directly. Dissipative mechanisms must be included to describe steady flow and yield phenomena. The microscopic model employed in this article is highly idealized, and should be expanded to include such features as more realistic structures and electrostatic 
multiples. Finally, this continuum approach can also be exploited to describe magnetorheological systems. Effects of magnetic saturation must be added to the development presented here.

\section{ACKNOWLEDGMENTS}

The authors thank V. Naletova, L. C. Davis, and A. J. Giacomin for helpful discussions. This work was supported in part by the National Science Foundation under Grant No. CTS-9502276.

\section{APPENDIX A: THE PHENOMENOLOGICAL ELECTROSTRICTION TENSORS}

The electrostriction tensors $\alpha_{i k l m}$ and $\gamma_{i k l m}$, defined in Eq. (7), each contain in general 81 components. Symmetry features reduce the total number of independent components. Here we outline the reduction of Eq. (7) to Eq. (9) for a transverse isotropic material possessing the distinct direction $\mathbf{d}$.

Following Sedov (1971), we decompose the electrostriction tensors in terms of the unit tensor and even polyadics of $\mathbf{d}$

$$
\begin{aligned}
\alpha_{i k l m}= & r_{1} \delta_{i k} \delta_{l m}+r_{2} \delta_{i l} \delta_{k m}+r_{3} \delta_{i m} \delta_{k l}+r_{4} \delta_{i k} d_{l} d_{m}+r_{5} \delta_{i l} d_{k} d_{m}+r_{6} \delta_{i m} d_{k} d_{l}+r_{7} \delta_{k l} d_{i} d_{m} \\
& +r_{8} \delta_{k m} d_{i} d_{l}+r_{9} \delta_{l m} d_{i} d_{k}+r_{10} d_{i} d_{k} d_{l} d_{m},
\end{aligned}
$$

where the scalars $r_{n}$ are material properties. An analogous expression exists for the tensor $\gamma_{i k l m}$. Only even polyadics appear because the electrostriction tensor must be invariant to a change in the sign of $\mathbf{d}$. The above expression can be reduced further by noting the symmetries

$$
\epsilon_{i k}=\epsilon_{k i}, \quad u_{l m}=u_{m l}, \quad \omega_{l m}=-\omega_{m l},
$$

which leads to Eq. (9) after relabeling the coefficients that remain. This reduction is consistent with the contracted notation presented by Mason (1958) for transverse isotropic materials.

\section{References}

Adriani, P. M. and A. P. Gast, “A microscopic model of electrorheology,’’ Phys. Fluids 31, 2757-2768 (1988). Bonnecaze, R. T. and J. F. Brady, "Dynamic simulation of an electrorheological fluid,' J. Chem. Phys. 96, 2183-2202 (1992).

Brooks, D., J. Goodwin, C. Hjelm, L. Marshall, and C. Zukoski, "'Visco-elastic studies on an electrorheological fluid,' Colloids Surface 18, 293-312 (1986).

Davis, L. C., "Polarization forces and conductivity effects in electrorheological fluids,' J. Appl. Phys. 72, 1334-1340 (1992).

Davis, L. C. and J. M. Ginder, "Electrostatic forces in electrorheological fluids,' in Proceedings of the Electrorheological Materials and Fluids Symposium, Washington D.C., August 21-22, 1994, edited by F. E. Filisko and K. O. Havelka (Plenum, New York, 1995), pp. 107-114.

Halsey, T. C., “Electrorheological fluids,'” Science 258, 761-766 (1992).

Heyes, D. M. and J. R. Melrose, "Brownian dynamics simulations of electro-rheological fluids. II: Scaling laws," Mol. Simul. 5, 293-306 (1990).

Jeffrey, D. J., "Conduction through a random suspension of spheres," Proc. R. Soc. London, Ser. A 335, 355-367 (1973).

Klingenberg, D. J., F. van Swol, and C. F. Zukoski, "The small shear rate response of electrorheological suspensions. I. Simulation in the point-dipole limit,' J. Chem. Phys. 94, 6160-6169 (1991).

Kraynik, A. M., R. T. Bonnecaze, and J. F. Brady, "Electrically induced stresses in ER fluids: The role of particle chain structure,', in Electrorheological Fluids, Mechanisms, Properties, Structure, Technology, and 
Applications, edited by R. Tao, Proceedings of the International Conference on Electrorheological Fluids, Carbondale, IL, October 15-16, 1991 (World Scientific, Singapore, 1992), pp. 59-74.

Landau, L. D. and E. M. Lifshitz, Electrodynamics of Continuous Media (Pergamon, New York, 1984).

Lemaire, E., G. Bossis, and Y. Grasselli, "Rheological behavior of electrorheological fluids," Langmuir 8, 2957-2961 (1992).

Martin, J. E. and R. A. Anderson, "Chain model of electrorheology,” J. Chem. Phys. 104, 4814-4827 (1996).

Mason, W. P., Physical Acoustics and the Properties of Solids (van Nostrand, Princeton, 1958).

McLeish, T. C. B., T. Jordan, and M. T. Shaw, "Viscoelastic response of electrorheological fluids. I. Frequency dependence,'” J. Rheol. 35, 427-448 (1991).

Parthasarathy, M. and D. J. Klingenberg, “Electrorheology: Mechanisms and models,” Mater. Sci. Eng. Rep. R17, 57-105 (1996).

Rosensweig, R. E., “On magnetorheology and electrorheology as states of unsymmetric stress," J. Rheol. 39, 179-192 (1995).

Russel, W. B., D. A. Saville, and W. R. Schowalter, Colloidal Dispersions (Cambridge University Press, Cambridge, 1989).

Sanfeld, A., Thermodynamics of Charged and Polarized Layers (Wiley, New York, 1968).

Sedov, L. I., A Course in Continuum Mechanics (Wolters-Noordhoff, Groningen, 1971), Vol. I.

Shkel, Y. M. and D. J. Klingenberg, "Material parameters for electrostriction,” J. Appl. Phys. 80, 4566-4572 (1996).

Shkel, Y. M. and D. J. Klingenberg, "Electrostriction of polarizable materials: Comparison of models with experimental data," J. Appl. Phys. 83, 7834-7843 (1998).

Stratton, J. A., Electromagnetic Theory (McGraw-Hill, New York, 1941).

Tamura, H., H. See, and M. Doi, "Model of porous particles containing water in electrorheological fluids," J. Phys. D 26, 1181-1187 (1993).

Tao, R. and J. M. Sun, “Ground state of electrorheological fluids from Monte Carlo simulations," Phys. Rev. A 44, R6181-R6184 (1991).

Tao, R. and Q. Jiang, "Simulation of structure formation in an electrorheological fluid," Phys. Rev. Lett. 73, 205-208 (1994).

Whittle, M., “Computer simulation of an electrorheological fluid," J. Non-Newtonian Fluid Mech. 37, 233263 (1990).

Xu, B. C. and K. C. Hass, “Liquid-state properties of electrorheological fluids," J. Chem. Phys. 98, 2258-2262 (1993). 